\title{
Silencing of c-kit with small interference RNA attenuates inflammation in a murine model of allergic asthma
}

\author{
WEI WU, TAO WANG, JIA-JIA DONG, ZENG-LIN LIAO and FU-QIANG WEN \\ Division of Pulmonary Diseases, State Key Laboratory of Biotherapy of China, and Department of Respiratory \\ and Tuberculosis Medicine, West China Hospital, Sichuan University, Chengdu, Sichuan 610041, P.R. China
}

Received January 15, 2012; Accepted March 9, 2012

DOI: $10.3892 / \mathrm{ijmm} .2012 .963$

\begin{abstract}
Asthma is a chronic respiratory disease characterized by the inflammation of the airways due to infiltration and activation of several inflammatory cells that produce cytokines. c-kit, a proto-oncogene that encodes a tyrosine kinase receptor, has been found to be associated with allergic inflammation. The aim of the present study was to assess whether silencing of c-kit with small interference RNA (siRNA) would attenuate inflammation in allergic asthma. A mouse model of ovalbumin (OVA)-induced allergic asthma was treated with systemic administration of anti-c-kit siRNA to inhibit the expression of the c-kit gene. siRNAs were injected through the vena caudalis. We measured inflammatory response in both anti-c-kit siRNA-treated and control mice. Systemic administration of siRNA could effectively inhibit the expression of the c-kit gene and reduce the infiltration of inflammatory cells (eosinophils and lymphocytes) into the lung tissue and bronchoalveolar lavage fluid. In addition, we found that c-kit siRNA can decrease the production of the T-helper type 2 (Th2) cytokines, interleukin 4 (IL-4) and IL-5, but has no influence on IFN- $\gamma$ generation. These results show that inhibition of c-kit expression with siRNA can reduce the inflammatory response in allergic asthma.
\end{abstract}

\section{Introduction}

Asthma is a chronic respiratory disease characterized by inflammation of the airways due to infiltration and activation of several inflammatory cells (mast cells, eosinophils, and T lymphocytes), which produce cytokines and proinflammatory molecules such as leukotrienes, interleukins, and chemotactic factors $(1,2)$. Antigen-presenting cells (APCs), especially dendritic cells, initiate and regulate the allergic responses

Correspondence to: Professor Fu-Qiang Wen, Department of Respiratory Medicine, West China Hospital, West China Medical School, Sichuan University, Chengdu, Sichuan 610041, P.R. China E-mail:wwatp@yahoo.cn; wenfuqiang@126.com

Key words: small interference RNA, c-kit, inflammation, asthma of T-helper type 2 (Th2) cells to allergens in asthma. APCs present allergens that are taken up and processed by Th2 cells, which leads to Th 2 cell activation. Activated Th2 cells produce a series of cytokines, such as interleukin 4 (IL-4), IL-5 and IL-13, which play a prominent role in disease pathogenesis by inducing the survival and recruitment of eosinophils and mast cells, by inducing goblet cell hyperplasia and bronchial hyperreactivity (3).

RNA interference (RNAi) is an innate mechanism in most eukaryotes, in which double-stranded RNA inside the cell targets the degradation of cognate mRNA in a sequencespecific manner (4). In recent years, in vivo small interference RNA (siRNA) delivery has been used to study specific gene functions and satisfactory results have been achieved (5).

The proto-oncogene c-kit is a membrane tyrosine kinase receptor encoded at the white spotting locus (W) and its ligand is stem cell factor (SCF). $c$-kit is critical for the proliferation, survival and differentiation of hematopoietic stem and progenitor cells and several non-hematopoietic tissues (6). A recent study showed that the inflammation in allergic asthma is alleviated in c-kit-defective mice, which indicates that c-kit plays a role in the development of allergic inflammation (7).

However, whether RNAi can attenuate the inflammation of allergic asthma mediated by c-kit is unknown. In this study, we used specific siRNA to silence the expression of the c-kit gene and investigated the effect of $c$-kit on allergic airway inflammation in an experimental model of asthma. We found that siRNA could inhibit the development and promote the resolution of airway inflammation.

\section{Materials and methods}

Experimental asthma model. Six to eight-week-old SPF-grade male C57BL/6 mice (20-25 g) were purchased from the Laboratory Animal Center of West China Hospital, Sichuan University. Experimental protocols were approved by the Institutional Animals Ethics Committee of West China School of Medicine, Sichuan University.

The experimental model of asthma was generated as previously described (8). Briefly, mice were sensitized by administering ovalbumin (OVA, $20 \mu \mathrm{g}$, grade V; SigmaAldrich) with aluminum hydroxide (alum, $2.25 \mathrm{mg}$; Imject Alum; Pierce Biotechnology, Inc.) in a $100 \mu \mathrm{l}$ total volume via intraperitoneal (i.p.) injection on Days 0 and 7. Mice were 
Table I. Characteristics of c-kit and scrambled siRNA.

\begin{tabular}{lcll}
\hline Target & Accession no. & & \multicolumn{1}{c}{ Sequence 5' $\rightarrow 3^{\prime}$} \\
\hline c-kit siRNA-1 & NM_021011 & Sense: & 5'-CUGUCUAGAAUUUACUCAAdTd-3' \\
& & Antisense: & 5'-UUGAUGAAAUUCUAGACAGdTdG-3' \\
c-kit siRNA-2 & NM_021011 & Sense: & 5'-CCG UGACAUUCAACGUUUAdTdT-3' \\
Scrambled siRNA & - & Antisense: & 5'-UAAACGUUGAAUGUCACGGdAdA-3' \\
& & Sense: & 5'-UAGGCGCAGCUCCGGAUCGdTT-3' \\
& & Antisense: & 5'-CGAUCCGGAGCUGCGCCUAdTT-3 \\
\hline
\end{tabular}

challenged with 5 doses of aerosolized 1\% OVA, each for 30 min, on Days 14-18. Mice were sacrificed with a lethal dose of pentobarbital via i.p. injection on Day 21.

Screening and chemical modification of the siRNA sequence. The siRNA sequence used for targeted silencing of c-kit [GenBank accession no. NM_021011] was designed by Qiagen, and siRNA sequences were selected according to the method of Sikarwar and Reddy (9). In addition, target siRNA was chemically modified by methylation to avoid or lessen its destruction in the blood (10). We selected two c-kit-specific siRNA duplexes on the basis of a previous in vitro study and used them as a mixture consisting of an equal amount of each siRNA duplex (9). We also designed a pair of control siRNAs, namely, scrambled siRNA and the sequence of scrambled siRNA with no complementation with any other genes in mice, which aims to avoid specific gene silencing. All the siRNAs were synthesized by Qiagen. Briefly, for each oligonucleotide, the 2 individual complementary strands of the siRNA were synthesized separately by the solid-phase method and then purified separately by ion-exchange chromatography. The complementary strands were annealed to form the doublestranded siRNA (duplex). The duplex was then ultrafiltered and lyophilized to form the solid drug substance (Table I).

siRNA knockdown of mouse c-kit in vivo. Mice were placed in a metal restrainer. Their tails were warmed by immersing in warm water $\left(45^{\circ} \mathrm{C}, 1 \mathrm{~min}\right)$ to dilate the blood vessels and were swabbed with alcohol. With the tail stretched, a hypodermic needle (30G) was inserted approximately parallel to the lateral tail vein. Appropriate needle placement was ensured by inserting the needle at least $3 \mathrm{~mm}$ into the vein lumen. The intravenous (i.v.) administration of siRNA was performed with caution to avoid rupture of the blood vessels during injection. The mice were divided into 3 groups of 5 mice each. The first group of asthmatic mice was injected with $0.1 \mathrm{ml}$ of PBS vehicle. The second and third groups of mice were injected with 5,10 or $20 \mu \mathrm{g}$ of specific siRNA for murine c-kit and scrambled siRNA for 3 consecutive days from Days 16 to 18 , respectively. The lung tissue and bronchoalveolar lavage fluid (BALF) were collected $72 \mathrm{~h}$ after the last injection.

Counting of inflammatory cells in the BALF. Bronchoalveolar lavage (BAL) with $1 \mathrm{ml}$ of HBSS, instilled bilaterally with a syringe, provided lavage fluid, which was harvested by gentle aspiration 3 times and then centrifuged (11). The cells in the
BALF were resuspended in PBS and counted with a hemocytometer. The cells were cytocentrifuged using Cytospin and then stained with Wright-Giemsa stain (Sigma) for counting of inflammatory cell subsets (eosinophils, alveolar macrophages and lymphocytes); 200 or more cells per slide were counted.

Enzyme-linked immunosorbent assay. The levels of inflammatory cytokines in the BALF were analyzed by enzyme-linked immunosorbent assays (ELISA), using ELISA kits for IL-4, IL-5, IFN- $\gamma$ and IL-6 (R\&D Systems, Inc., USA) according to the manufacturer's instructions. Five mice were used for each group per experiment.

Histology. Lung tissue blocks from the right upper and right middle lobes were rinsed in normal saline and fixed in $10 \%$ formalin for $24 \mathrm{~h}$. The specimens were then embedded in paraffin after being dehydrated through ascending grades of ethanol and cleared in toluene. The tissue blocks were sectioned at $4 \mu \mathrm{m}$ with a Leica rotary microtome. Paraffin sections were mounted on albuminized glass slides by floating and flattening the sections in a water bath at $45^{\circ} \mathrm{C}$. The mounted sections were drained until dry and kept in an incubator at $30^{\circ} \mathrm{C}$. Paraffin sections were first dewaxed in 2 changes of xylene, then passed through descending grades of alcohol, and finally washed in deionized water before staining with hematoxylin and eosin for $1 \mathrm{~min}$ at room temperature. The sections were rinsed 3 times in deionized water, then quickly dehydrated through ascending grades of ethanol, and passed through xylene before being mounted with Permount; the sections were then photographed and evaluated. The paraffin sections were stained with hematoxylin and eosin and periodic acid-Schiff reagent (Sigma).

$R T$-PCR for $c$-kit. For RNA isolation, the whole left lungs were frozen in liquid nitrogen, stored at $-80^{\circ} \mathrm{C}$ and frozen immediately after removal from mice in each group. Total-RNA was extracted from frozen lung tissue using TRIzol reagent (Gibco-BRL, Gaithersburg, MD) and amplified using the Promega PCR single-step kit (Promega, Madison, WI), according to the manufacturer's instructions. Total-RNA was transcribed into cDNA with reverse transcriptase kit at $50^{\circ} \mathrm{C}$ for $30 \mathrm{~min}$. The following primers for murine c-kit were used: forward, 5'-ACCCACAGGTGTCCAATTATTC-3' and reverse, 5'-TGG CGTTCATAATTGAAGTCAC-3'. $\beta$-actin served as the internal control. The PCR products were electrophoresed on a $3 \%$ agarose gel and visualized by ethidium bromide staining. 

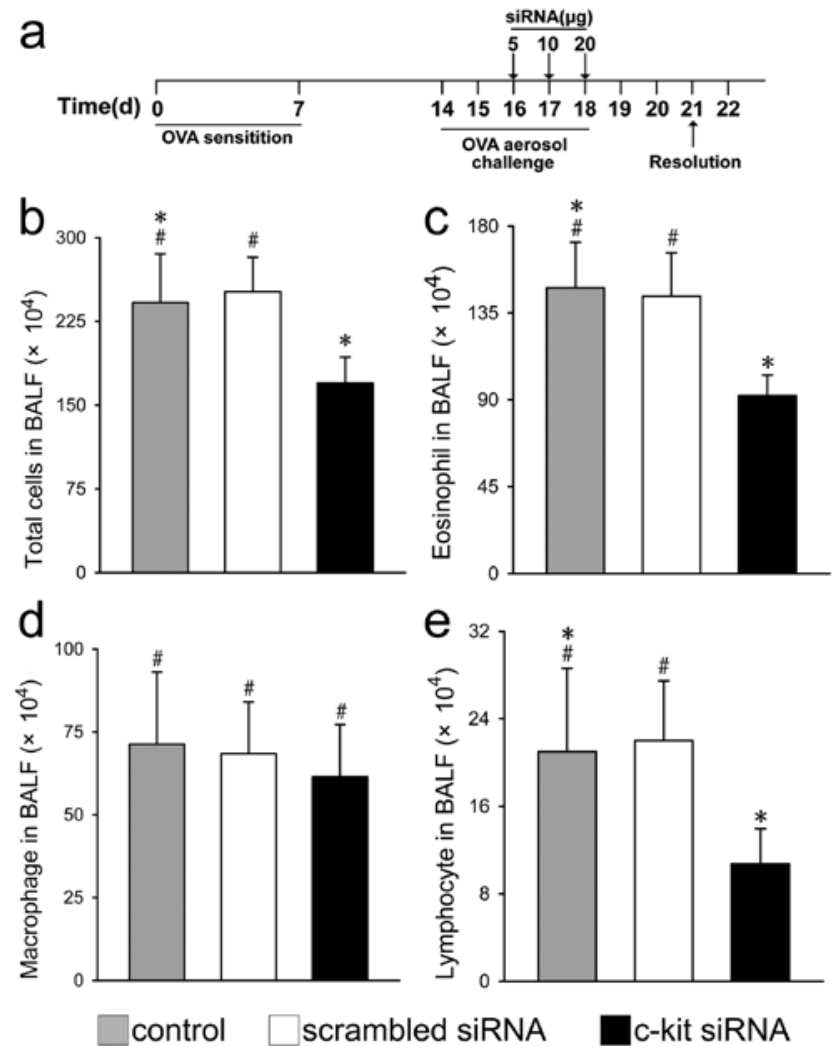

Figure 1. siRNA/c-kit dampens the development of allergic airway inflammation. (a) Protocol to induce allergic airway inflammation: mice were sensitized with OVA (intraperitoneally) on Days 0 and 7 and challenged with aerosolized OVA on Days 14-18. The siRNAs were injected into the mice through the vena caudalis at doses of 5,10 and $20 \mu \mathrm{g}$, respectively, on Days 16-18. Samples were obtained 3 days after the injection of siRNA or PBS vehicle,on Day 21. Number of (b) total cells (c) eosinophils, (d) alveolar macrophages and (e) lymphocytes in BALF. ${ }^{~} \mathrm{P}<0.05$; ${ }^{*} \mathrm{P}>0.05$ (Student's $\mathrm{t}$-test). Data (mean $\pm \mathrm{SEM}$ ) are representative of three independent experiments with $\geq 5$ mice.

Western blotting. Tissue blocks from the left lobe of the lung were immediately immersed in liquid nitrogen. The tissues were ground and then lysed in RIPA lysis buffer. Lysate $(40 \mu \mathrm{g})$ was loaded per well and resolved on a $10 \%$ polyacrylamide gel (SDS-PAGE) under denaturing conditions; the proteins were transferred onto $0.45 \mu \mathrm{m}$ nitrocellulose membranes. The blots were probed for murine c-kit using a murine c-kit antibody (Santa Cruz Biotechnology, Inc.); they were also probed with the GAPDH antibody as a loading control (Santa Cruz Biotechnology, Inc.). Bands were visualized using an antirabbit IgG-HRP-conjugated secondary antibody and the ECL western blotting detection system (GE Healthcare, UK).

Statistical analysis. The results are expressed as the mean \pm SD, unless indicated otherwise. Statistical analysis was performed by the SPSS software using one-way analysis of variance (ANOVA), followed by LSD significant difference test. A value of $\mathrm{P}<0.05$ (two-tailed test) was considered statistically significant.

\section{Results}

siRNA attenuates the inflammatory response in the BALF. All BALF samples were obtained 3 days after the injection
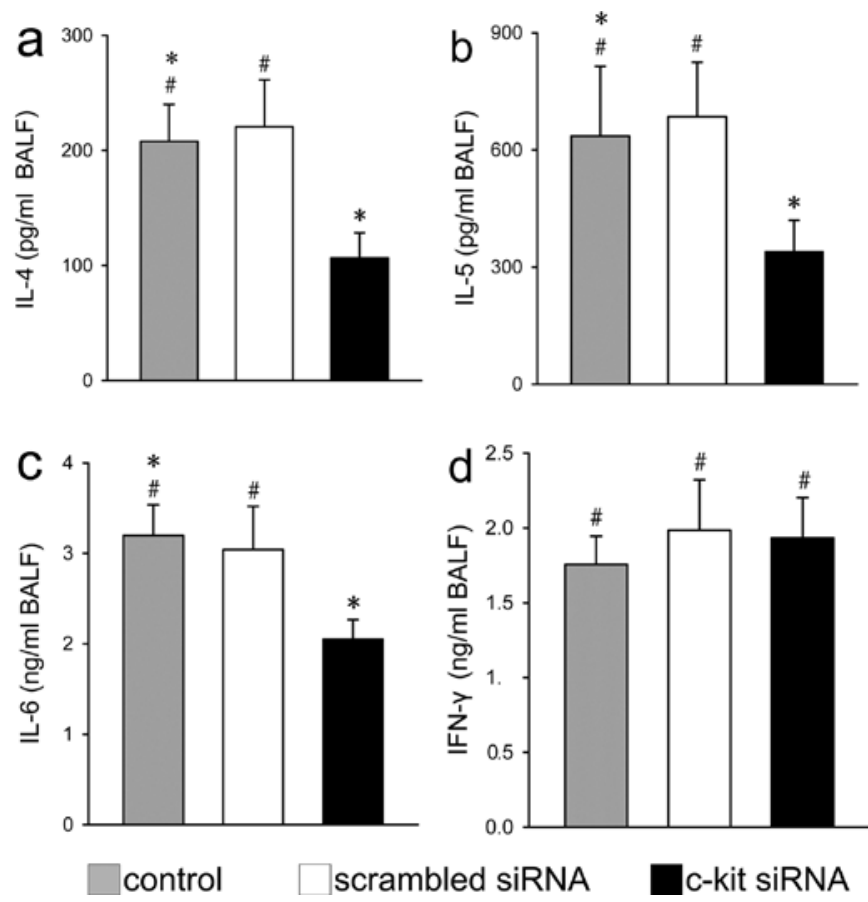

Figure 2. The anti-c-kit siRNA selectively regulates cytokines in BALF. Samples were obtained 3 days after the injection of siRNA or PBS vehicle, on Day 21. The levels of (a) IL-4, (b) IL-5, (c) IL-6, (d) IFN- $\gamma$ of three groups in BALF, were measured by ELISA. BALF was collected at $72 \mathrm{~h}$ after the last administration. ${ }^{*} \mathrm{P}<0.05$; ${ }^{\text {"}} \mathrm{P}>0.05$ (Student's t-test). Data (mean $\pm \mathrm{SEM}$ ) are representative of three independent experiments with $\geq 5$ mice.

of siRNA or PBS vehicle on Day 21. The number of total cells, eosinophils and lymphocytes in BALF decreased more significantly in the c-kit siRNA group than in the control and scrambled-siRNA groups $(\mathrm{P}<0.05)$ (Fig. 1b, c and e). The number of macrophages in the c-kit-siRNA group was lower than those in the control or the scrambled-siRNA group, but the difference was not significant $(\mathrm{P}>0.05)$ (Fig. 1d).

c-kit siRNA selectively regulates the production of cytokines in the BALF. The production of the Th2 cytokines IL-4 and IL-5 decreased to a greater extent in the c-kit siRNA group than in the control and scrambled-siRNA groups (Fig. 2a and b); the same pattern was observed in the production of IL-6 (Fig. 2c). The production of IFN- $\gamma$, a Th1 cytokine, did not increase with the decrease in Th2 cytokines (Fig. 2d).

siRNA attenuates the inflammatory response of lung tissue and reduces the secretion of mucus in the airways of asthmatic mice. Histological examination of lung specimens revealed that c-kit siRNA attenuated the inflammatory response to a greater extent in the lung tissue than in the control and scrambledsiRNA groups. Compared with the control group, the number of inflammation cells that infiltrated in lung tissue was lower in the c-kit siRNA group (Fig. 3).

c-kit siRNA can downregulate the expression of c-kit gene in lung tissue. To investigate whether c-kit-specific siRNA could silence the expression of the c-kit gene, we detected levels of the c-kit mRNA by reverse transcription-polymerase chain reaction (RT-PCR). In comparison with the control group and 


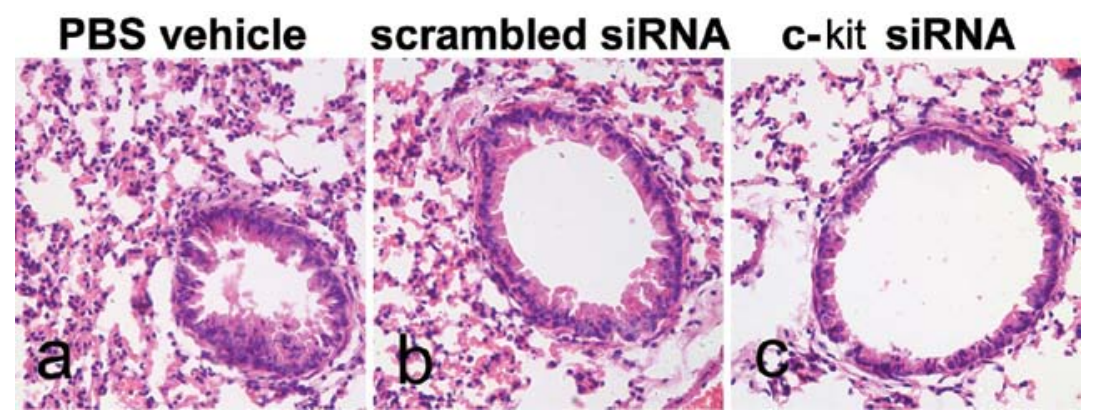

Figure 3. Histological study of the asthmatic lung and airway inflammation and its suppression by anti-c-kit siRNA. Lung tissue sections at Day 21 from mice given siRNA or vehicle, obtained from fixed, paraffin-embedded lung tissue stained with hematoxylin and eosin. Original magnification $\mathrm{x} 400$. The mice were injected with (a) PBS vehicle, (b) scrambled siRNA and (c) c-kit siRNA through caudal vein. The experiment was carried out thrice and representative images are shown.
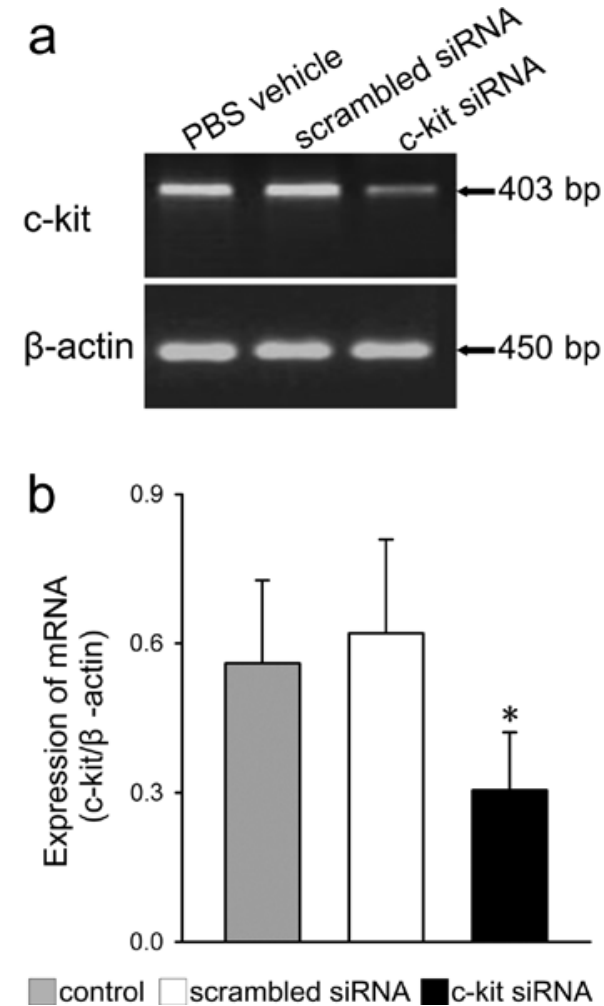

Figure 4. Representative RT-PCR analysis of c-kit mRNA expression in lung tissue. (a) Note that c-kit mRNA expression was downregulated at $72 \mathrm{~h}$ after the last administration in the anti-c-kit siRNA group. (b) The bargraph represent mean value $\pm \mathrm{SD}$ of the relative intensity of the c-kit bands normalized to $\beta$-actin. "P $<0.05$ compared with control and scrambled-siRNA group. The experiment was carried out thrice and representative images are shown.

scrambled siRNA group, the result showed that the mRNA expression of c-kit mRNA was downregulated in the anti-ckit siRNA group at $72 \mathrm{~h}$ after the last administration, which indicated that anti-c-kit siRNA can effectively inhibit the expression of the c-kit gene (Fig. 4).

Western blot analysis indicates that c-kit siRNA inhibits the expression of c-kit protein. Western blot analysis showed that anti-c-kit siRNA could effectively inhibit the expression of c-kit protein at $72 \mathrm{~h}$ post-transfection in the c-kit siRNA group; in contrast, the control and scrambled-siRNA groups showed expression of c-kit protein in lung tissue (Fig. 5).
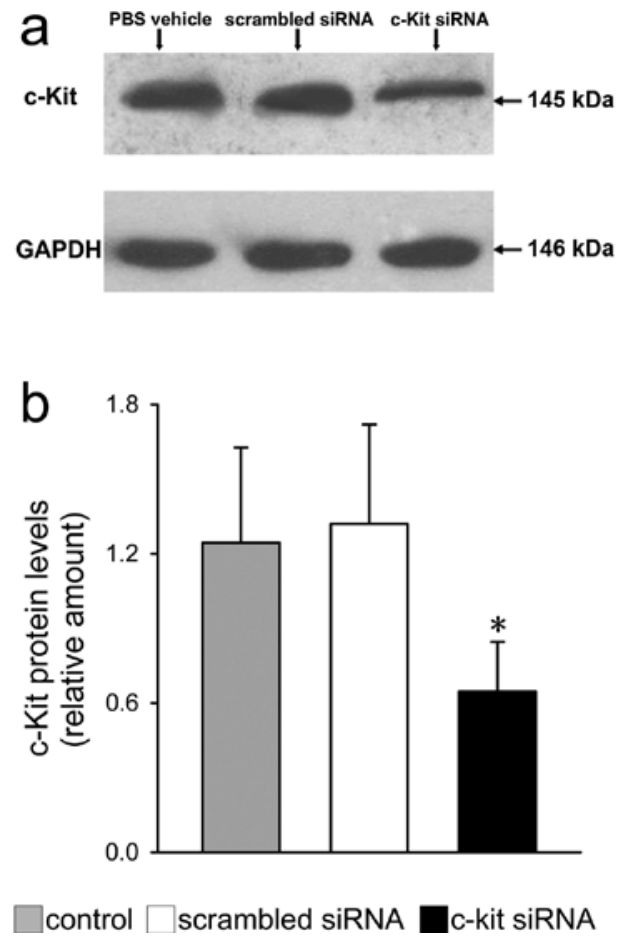

Figure 5. (a) Expression of c-kit protein in lung tissue. (b) Histograms represent mean value $\pm \mathrm{SD}$ of the relative amount of the c-kit bands normalized to GAPDH. There was a difference between the anti-c-kit siRNA group and the other groups. ${ }^{*} \mathrm{P}<0.05$ vs. the control group and the scrambled-siRNA group. The experiment was carried out thrice and representative images shown.

\section{Discussion}

In the majority of eukaryotes, RNAi is an evolutionarily conserved process that inhibits gene expression primarily by targeting mRNAs (12). To date, numerous studies have been conducted in a variety of animal models to investigate the efficacy of siRNAs as therapeutic agents; their findings indicate that specific siRNAs could inhibit the expression of target genes when administered locally or systemically $(13,14)$. In this study, we used a mouse model of allergic asthma; the mice were systematically administered c-kit siRNA by i.v. injection. We obtained evidence that c-kit siRNA acts as an anti-inflammatory and pro-resolution mediator of allergic airway inflammation. 
The c-kit proto-oncogene has been found to be expressed in a wide range of non-hematopoietic cell types (with the exception of hematopoietic stem and/or progenitor mast cells), including vascular endothelial cells and interstitial cells of Cajal, which control gut motility, astrocytes, renal tubules, breast glandular epithelial cells and sweat glands (15-19). A few studies have shown high cell-surface expression of c-kit in the human airway epithelia of asthmatic patients $(20,21)$.

$\mathrm{c}-\mathrm{kit}$ is a member of the PDGF family of receptor tyrosine kinases and is the ligand of the stem cell factor (SCF). The c-kit receptor is present in mast cells, eosinophils and lymphocytes (22). These cells play an important role in the development of asthmatic airway inflammation (23). In our study, we used specific siRNA to inhibit the expression of c-kit and found that it could attenuate inflammation in allergic asthma. The number of total cells, eosinophils and lymphocytes markedly decreased in the BALF from the mice administered c-kit siRNA. Histopathologic examination of the lung showed that treatment with c-kit siRNA reduced the inflammation of lung tissue in the airways. The c-kit ligand or SCF has been identified as a primary inducer of mast cell differentiation, proliferation and activation. Blocking c-kit/c-kit ligand interaction could significantly decrease eosinophils in BALF (24). Some studies have shown that c-kit/SCF enhances IgE-dependent mediator release from mast cells, such as histamine and leukotrienes $(24,25)$. c-kit/SCF is closely related to airway infiltration of mast cells and eosinophils which promotes degranulation of mastocytes. Our study showed that anti-c-kit siRNA could effectively downregulated c-kit expression, which contribute to reducing allergic inflammation mediated by c-kit in mice.

Four isoforms of c-kit in humans have been identified, which result from alternate mRNA splicing. Each isoform differs in signaling and function (26). Previous studies have shown that the function of c-kit is induced by the activation of several different signaling pathways, including MAPK, PI-3 kinase and the small GTPase Rac, which are independent pathways that interact in signal transduction (6). The c-kit-PI3 kinase signaling axis positively regulates the production of IL-6; moreover, c-kit is associated with Th2 responses through the Notch ligand Jagged-2 signaling pathway $(7,27)$. IL-6 has been associated with Th2 and Th17 differentiation (28). In our study, the production of IL-6 in the BALF decreased to a markedly greater extent in the c-kit siRNA group than in the control group, which suggests that PI-3 kinase plays a role in regulating IL-6 production. Therefore, we hypothesized that the Notch signaling pathway is involved in the production of Th2 cytokines (IL-4 and IL-5) through Th2 cell polarization mediated by c-kit. Furthermore, c-kit upregulation on dendritic cells led to immune skewing toward $\mathrm{T}$ helper Th2 and Th17 subsets and away from Th1 responses (29). Silencing of the c-kit gene with specific siRNA suppressed the production of IL- 6 and Th2 and Th17 responses, which attenuated the allergic inflammation mediated by c-kit. On the other hand, inhibition of Th2 response results in the decrease of IL-4 and IL-5 production.

IL-4 and IL-5, which are Th2 cytokines, play an important role in the development of allergic asthma. Previous studies using a murine model of asthma have shown that treatment with antisense phosphorothioate oligonucleotides to the c-kit ligand suppresses airway inflammation, IL-4 production and eosinophilia (30). In addition, IL-4 promotes IgE synthesis, contributes to early recruitment of eosinophils and is detected as early as $3 \mathrm{~h}$ after challenge (31-33). Additionally, IL-4 production leads to production of IL-5 and other cytokines in asthma (34). IL-5 is the most important chemokine in the recruitment of eosinophils into the airway. In our study, we found that the levels of IL-4, IL-5 and IL-6 decreased in the BALF from the mice treated with c-kit siRNA, which suggests that activation of c-kit could promote the production of IL-4, and that IL-4 plays a pivotal role in asthma pathogenesis. Thus, c-kit siRNA inhibits c-kit activation, which results in a decrease in IL-4 production and, consequently, a lower production of IL-5; this leads to diminished recruitment of eosinophils into the airway and, ultimately, to a decrease in the number of eosinophils in the BALF. In addition, we found that the number of lymphocytes decreased in the BALF from the group treated with c-kit siRNA; however, the mechanism of this effect is unclear.

In conclusion, our data show that c-kit-specific siRNA attenuates inflammation in a mouse model of OVA-induced allergic asthma through inhibiting the production of inflammatory cytokines. We have shown that systemically administered siRNA can induce target gene silencing, which indicates the potential use of c-kit siRNA in the treatment of asthma.

\section{Acknowledgements}

This study was supported by grants 30971327 and 31171103 from National Natural Science Foundation of China and grant 06-834 from China Medical Board of New York to Dr F.Q. Wen.

\section{References}

1. Martinez Molina D, Wetterholm A, Kohl A, McCarthy AA, Niegowski D, Ohlson E, Hammarberg T, Eshaghi S, Haeggström JZ and Nordlund P: Structural basis for synthesis of inflammatory mediators by human leukotriene $C 4$ synthase. Nature 448: 613-616, 2007.

2. Holgate ST: The epidemic of allergy and asthma. Nature 402: B2-B4, 1999.

3. Hammad H and Lambrecht BN: Dendritic cells and epithelial cells: linking innate and adaptive immunity in asthma. Nat Rev Immunol 8: 193-204, 2008.

4. Ovcharenko D, Jarvis R, Hunicke-Smith S, Kelnar K and Brown D: High-throughput RNAi screening in vitro: from cell lines to primary cells. RNA 11: 985-993, 2005.

5. Xie FY, Woodle MC and Lu PY: Harnessing in vivo siRNA delivery for drug discovery and therapeutic development. Drug Discov Today 11: 67-73, 2006.

6. Edling CE and Hallberg B: c-kit - a hematopoietic cell essential receptor tyrosine kinase. Int J Biochem Cell Biol 39: 1995-1998, 2007.

7. Krishnamoorthy N, Oriss TB, Paglia M, Fei M, Yarlagadda M, Vanhaesebroeck B, et al: Activation of c-kit in dendritic cells regulates $\mathrm{T}$ helper cell differentiation and allergic asthma. Nat Med 14: 565-573, 2008.

8. Paveglio SA, Allard J, Mayette J, Whittaker LA, Juncadella I, Anguita $J$ and Poynter ME: The tick salivary protein, Salp15, inhibits the development of experimental asthma. J Immunol 178: 7064-7071, 2007.

9. Sikarwar AP and Reddy KV: siRNA-mediated silencing of c-kit in mouse primary spermatogonial cells induces cell cycle arrest. Oligonucleotides 18: 145-160, 2008.

10. Strapps WR, Pickering V, Muiru GT, Rice J, Orsborn S, Polisky BA, Sachs A and Bartz SR: The siRNA sequence and guide strand overhangs are determinants of in vivo duration of silencing. Nucleic Acids Res 38: 4788-4797, 2010. 
11. Oriss TB, Ostroukhova M, Seguin-Devaux C, Dixon-McCarthy B, Stolz DB, Watkins SC, Pillemer B, et al: Dynamics of dendritic cell phenotype and interactions with $\mathrm{CD}^{+} \mathrm{T}$ cells in airway inflammation and tolerance. J Immunol 174: 854-863, 2005.

12. Gondi CS and Rao JS: Concepts in in vivo siRNA delivery for cancer therapy. J Cell Physiol 220: 285-291, 2009.

13. Pushparaj PN, H'ng SC and Melendez AJ: Refining siRNA in vivo transfection: silencing SPHK1 reveals its key role in C5a-induced inflammation in vivo. Int J Biochem Cell Biol 40: 1817-1825, 2008.

14. Zhang W, Yang H, Kong X, Mohapatra S, San Juan-Vergara H, Hellermann G, Behera S, et al: Inhibition of respiratory syncytial virus infection with ntranasal siRNA nanoparticles targeting the viral NS1 gene. Nat Med 11: 56-62, 2005.

15. Broudy VC, Kovach NL, Bennett LG, Lin N, Jacobsen FW and Kidd PG: Human umbilical vein endothelial cells display highafinity c-kit receptors and produce a soluble form of the c-kit receptor. Blood 83: 2145-2152, 1994.

16. Torihashi S, Ward SM, Nishikawa S, Nishi K, Kobayashi S and Sanders KM: c-kit-dependent development of interstitial cells and electrical activity in the murine gastrointestinal tract. Cell Tissue Res 280: 97-111, 1995 .

17. Huizinga JD, Thuneberg L, Klüppel M, Malysz J, Mikkelsen HB and Bernstein A: W/kit gene required for interstitial cells of Cajal and for intestinal pacemaker activity. Nature 373: 347-349, 1995.

18. Natali PG, Nicotra MR, Sures I, Santoro E, Bigotti A and Ullrich A: Expression of c-kit receptor in normal and transformed human nonlymphoid tissues. Cancer Res 52: 6139-6143, 1992.

19. Lammie A, Drobnjak M, Gerald W, Saad A, Cote R and CordonCardo C: Expression of c-kit and kit ligand proteins in normal human tissues. J Histochem Cytochem 42: 1417-1425, 1994.

20. Al-muhsen SZ, Shablovsky G, Olivenstein R, Mazer B and Hamid Q: The expression of stem cell factor and c-kit receptor in human asthmatic airways. Clin Exp Allergy 34: 911-916, 2004.

21. Zhang Z, Zhang R, Joachimiak A, Schlessinger J and Kong XP: Crystal structure of human stem cell factor: implication for stem cell factor receptor dimerization and activation. Proc Natl Acad Sci USA 97: 7732-7737, 2000.

22. Broudy VC: Stem cell factor and hematopoiesis. Blood 90 1345-1364, 1997.

23. Rossi GL and Olivieri D: Does the mast cell still have a key role in asthma? Chest 112: 523-529, 1997.
24. Lukacs NW, Strieter RM, Lincoln PM, Brownell E, Pullen DM, Schock HJ, et al: Stem cell factor (c-kit ligand) influences eosinophil recruitment and histamine levels in allergic airway inflammation. J Immunol 156: 3945-3951, 1996.

25. Columbo M, Horowitz EM, Botana LM, MacGlashan DW Jr, Bochner BS, Gillis S, et al: The human recombinant c-kit receptor ligand, rhSCF, induces mediator release from human cutaneous mast cells and enhances IgE-dependent mediator release from both skin mast cells and peripheral blood basophils. J Immunol 149: 599-608, 1992.

26. Ashman LK: The biology of stem cell factor and its receptor c-kit. Int J Biochem Cell Biol 31: 1037-1051, 1999.

27. Amsen D, Blander JM, Lee GR, Tanigaki K, Honjo T and Flavell RA: Instruction of distinct CD4 T helper cell fates by different notch ligands on antigen-presenting cells. Cell 117: 515-526, 2004.

28. Dodge IL, Carr MW, Cernadas M and Brenner MB: IL-6 production by pulmonary dendritic cells impedes Th1 immune responses. J Immunol 170: 4457-4464, 2003.

29. Ray P, Krishnamoorthy N, Oriss TB and Ray A: Signaling of c-kit in dendritic cells influences adaptive immunity. Ann NY Acad Sci 1183: 104-122, 2010.

30. Finotto S, Buerke M, Lingnau K, Schmitt E, Galle PR and Neurath MF: Local administration of antisense phosphorothioate oligonucleotides to the c-kit ligand, stem cell factor,suppresses airway inflammation and IL-4 production in a murine model of asthma. J Allergy Clin Immunol 107: 279-286, 2001.

31. Corry DB, Folkesson HG, Warnock ML, Erle DJ, Matthay MA, Wiener-Kronish JP and Locksley RM: Interleukin 4, but not interleukin 5 or eosinophils, is required in a murine model of acute airway hyperreactivity. J Exp Med 183: 109-117, 1996.

32. Lukacs NW, Strieter RM, Chensue SW and Kunkel SL: Interleukin-4-dependent pulmonary eosinophil infiltration in a murine model of asthma. Am J Respir Cell Mol Biol 10: 526-532, 1994.

33. Ohkawara Y, Lei XF, Stampfli MR, Marshall JS, Xing Z and Jordana M: Cytokine and eosinophil responses in the lung, peripheral blood, and bone marrow compartments in a murine model of allergen-induced airways inflammation. Am J Respir Cell Mol Biol 16: 510-520, 1997.

34. Wills-Karp M: Murine models of asthma in understanding immune dysregulation in human asthma. Immunopharmacology 48: 263-268, 2000. 